\title{
The impact of ASCT on patients with newly diagnosed multiple myeloma who receive RVD induction
}

\author{
Marlise R. Luskin ${ }^{1}$, Federico Campigotto ${ }^{2}$, Paul G. Richardson ${ }^{3}$, John Koreth ${ }^{3}$, I rene M. Ghobrial ${ }^{3}$, \\ Robert L. Schlossman ${ }^{3}$, Nikhil C. Munshi ${ }^{3}$, Edwin P. Alyea ${ }^{3}$, Robert J. Soiffer ${ }^{3}$, Diane Warren $^{3}$, \\ Muriel Gannon ${ }^{3}$, Kathleen Finn ${ }^{3}$, Kenneth C. Anderson ${ }^{3}$, Edie A. Weller ${ }^{2}$, J acob P. Laubach ${ }^{3}$ \\ 1. Department of Medicine, Brigham and Women's Hospital, USA. 2. Department of Biostatistics and Computational \\ Biology, Dana-Farber Cancer Institute, USA. 3. Department of Medical Oncology, Dana-Farber Cancer Institute, USA \\ Correspondence: Jacob Laubach. Address: 450 Brookline Avenue, Boston, MA 02215, USA. \\ Email: jacobp_laubach@dfci.harvard.edu
}

Received: May 31, 2013

DOI : 10.5430/jhm.v3n1p41
Accepted: July 7, 2013

Online Published: July 12, 2013

\section{Abstract}

Objective: Lenalidomide-bortezomib-dexamethasone (RVD) is associated with a high-rate of response in newly diagnosed multiple myeloma (MM). Autologous stem cell transplantation (ASCT) with high-dose melphalan conditioning is a standard of care in MM. The impact of ASCT following RVD induction is not known. This study evaluates the impact of ASCT following RVD induction therapy in MM.

Methods: Using our center's transplant database, we identified 60 patients with newly diagnosed MM who underwent RVD induction followed by ASCT in first response. We describe disease characteristics including International Staging System (ISS) stage and cytogenetics, pre- and post-ASCT response, as well as progression-free survival (PFS) from time of ASCT.

Results: The rate of paraprotein complete response prior to and following ASCT was 50\% and 60\%, respectively. Among the 30 patients who did not achieve a paraprotein CR pre-ASCT, 14 (47\%) patients had improved response post-transplant. At a median follow-up of 23 months, 90\% (90\% CI: [81\%, 95\%]) of the patients were alive without progression 12 months after transplant.

Conclusion: ASCT improves the rate of CR in patients with newly diagnosed MM who receive RVD induction therapy.

\section{Key words}

Multiple myeloma, Induction therapy, Autologous stem cell transplantation

\section{I ntroduction}

Multiple myeloma (MM) is a B-cell neoplasm characterized by proliferation of clonal plasma cells in the bone marrow and extramedullary sites, and the associated clinical manifestations of lytic bone disease, hypercalcemia, renal impairment, and cytopenias ${ }^{[1]}$. Regimens incorporating the immunomodulatory agents thalidomide and lenalidomide, and/or the proteasome inhibitor bortezomib, are now important options for newly diagnosed disease ${ }^{[2-5]}$. 
The combination of an immunomodulatory agent and a proteasome inhibitor as induction therapy is associated with a high rate of overall and complete response ${ }^{[6,7]}$. The rationale for these combinations derives from in vitro models demonstrating synergistic anti-MM activity between the drug classes and an ability to overcome drug resistance ${ }^{[8]}$. In a phase I/II study evaluating lenalidomide-bortezomib-dexamethasone (RVD) in newly diagnosed disease, the overall response rate (ORR) was $100 \%$ including a rate of very good partial response (VGPR) or better of $67 \%$ and $74 \%$ in all patients and the phase II population respectively ${ }^{[7]}$.

Following induction therapy, patients who are transplant-eligible may undergo consolidation with high-dose melphalan and autologous stem cell transplantation (ASCT) with the aim of both deepening and prolonging response ${ }^{[9]}$. It is well documented that achievement of a complete remission (CR) both prior to ${ }^{[10,11]}$ and post-transplant ${ }^{[9,10,12-19]}$ correlates with longer progression-free and overall survival.

Whether ASCT can improve the depth of response associated with RVD, and specifically the rate of CR, is not known. In this study we report on the outcome among patients at our center with newly diagnosed MM who received RVD induction followed by ASCT.

\section{Methods}

Query of the hematologic malignancies transplant database and the electronic medical record (EMR) at the Dana Farber Cancer Institute (DFCI) identified 60 patients with newly diagnosed multiple myeloma (MM) whose induction treatment consisted of RVD, defined as receiving at least 2 cycles of RVD induction, followed by single autologous stem cell transplant with high-dose melphalan conditioning between January 1, 2005 and December 31, 2010. The classic RVD regimen consists of intravenous bortezomib $1.3 \mathrm{mg} / \mathrm{m}^{2}$ days $1,4,8$, and 11 ; lenalidomide 15 to $25 \mathrm{mg}$ days 1-14, and oral dexamethasone 20 or $40 \mathrm{mg}$ days 1, 2, 4, 5, 8, 9, and 11, 12 although strict adherence to the classic regimen could not be verified among patients who received induction therapy at sites other than the transplant center. Patients who discontinued no more than one of the induction agents due to toxicity were included in this analysis. Patients included in this analysis were deemed by their treating physician appropriate candidates for high-dose therapy and ASCT. All patients received high-dose melphalan $200 \mathrm{mg} / \mathrm{m}^{2}$ as conditioning prior to autologous stem cell rescue.

In addition to patients who received RVD throughout the course of induction therapy, patients who received RVD induction after no more than 2 initial cycles of either lenalidomide-dexamethasone (RD), bortezomib-dexamethasone (VD), or single agent dexamethasone (D) were included in the analysis. Such patients were included because for a variety of reasons it is relatively common in clinical practice outside the context of clinical trials for patients to receive one or two cycles of therapy with RD, VD, or D prior to conversion to RVD.

For the 60 eligible patients, demographic information (gender, age), disease sub-type (IgG, $\operatorname{IgA}$, light chain-only, or other) and risk profile, as assessed by the International Staging System (ISS) stage, were identified from the transplant database. Risk assessment as determined by metaphase cytogenetics and fluorescent in-situ hybridization (FISH) was identified from the EMR. High risk disease was defined as presence of del 13 (by metaphase), $\mathrm{t}(4 ; 14), \mathrm{t}(14 ; 16), \mathrm{t}(14 ; 20)$, del 17p, hypodiploidy and/or complex cytogenetics ${ }^{[20]}$.

Each patient's induction regimen was characterized, including the number of cycles of RVD induction. Toxicities requiring discontinuation of lenalidomide or bortezomib during RVD induction were described. The stem cell collection yield and time to hematopoietic recovery following ASCT was obtained from the transplant database. Platelet recovery was defined as the date when the platelet count reached $20,000 / \mathrm{mm}^{3}$ and was maintained at that level for two consecutive evaluations. Neutrophil recovery was defined as the date when the absolute neutrophil count (ANC) reached 500/mm and was maintained at that level for two consecutive evaluations. The EMR was reviewed to identify the presence or absence of lenalidomide maintenance post-transplant. 
Disease response prior to and post ASCT was evaluated using M-protein (or serum free light chain (FLC) when M-protein was not measurable or unavailable) and bone marrow plasma cell percentage. M-protein response was divided into 4 categories guided by International Myeloma Working Group (IMWG) response criteria ${ }^{[21]}$. A complete paraprotein response (paraprotein $\mathrm{CR}$ ) was defined as the absence of a paraprotein spike in the serum or urine by immunofixation (or normal FLC ratio). A very good paraprotein response (paraprotein VGPR) was defined as a $\geq 90 \%$ reduction in serum M-protein plus a urine M-protein of $<100 \mathrm{mg} / 24$ hours (or $\geq 90 \%$ reduction in difference between FLC levels). A partial paraprotein response (paraprotein PR) was defined as a $\geq 50 \%$ reduction of serum M-protein plus reduction in 24-hour urine M-protein by $\geq 90 \%$ or $<200 \mathrm{mg} / 24$ hours (or $\geq 50 \%$ reduction in difference between FLC levels). A stable paraprotein response (SD) was defined as a response not meeting criteria for a paraprotein CR, paraprotein VGPR, or paraprotein PR. Progressive disease (PD) was defined as an increase of $25 \%$ from lowest response of any measure of M-protein (serum M-protein must have increased by $\geq 0.5 \mathrm{~g} / \mathrm{dL}$, urine M-protein must have increased by $\geq 200 \mathrm{mg} / 24 \mathrm{~h}$, difference in FLC levels must have increased by $\geq 10 \mathrm{mg} / \mathrm{dL}$ ).

The median follow-up from ASCT at the time of this analysis is 22.6 (range: 7.6-50.6) months. The institutional review board at the transplant center approved this retrospective study.

\section{Statistical analysis methods}

Descriptive statistics are provided for all endpoints. Patient and disease characteristics, treatment course, best paraprotein response prior to and post ASCT (up to day 120 and prior to additional chemotherapy/maintenance treatment) are summarized using medians and ranges for continuous endpoints and frequencies, proportions and $95 \%$ exact binomial confidence intervals (CIs) for binary endpoints. Progression-free survival (PFS) is estimated using the Kaplan-Meier method ${ }^{[22]}$. PFS is defined as the time from the date of ASCT to the date of disease progression or death from any cause, whichever occurs first. The follow-up times for patients alive without progression are censored at the date last known to be free of disease progression. Univariate and multivariable Cox regression models were used to evaluate the effect of ISS and cytogenetic risk on PFS. The statistical analysis for this paper was performed using SAS/STAT software, Version 9.2 of the SAS System for Windows. Copyright (C) 2002-2008 SAS Institute Inc. SAS and all other SAS Institute Inc. product or service names are registered trademarks or trademarks of SAS Institute Inc., Cary, NC, USA.

\section{Results}

\subsection{Patient and disease characteristics}

Among the 60 patients (see Table 1), 39 (65\%) were male with median age at induction of 57 years (range 39 to 71 ). $\operatorname{IgG}$ was the most common subtype (50\% IgG, $21.7 \%$ IgA, $26.6 \%$ light chain disease, and $1.7 \%$ other). By ISS stage, 48,30 , 17 , and $5 \%$ had stage I, II, III, and unknown disease. Based on cytogenetics, 63 , 25, and $12 \%$ had standard, high, and unknown-risk disease.

\subsection{Pre-ASCT response}

Patients received a median of 5.5 cycles of RVD (range 2 to 13, interquartile range 4-7 cycles, Table 1). Among the 60 patients, 15 (25\%) patients received 1 or 2 cycles of VD, RD or dexamethasone alone prior to RVD (11 patients had 1 cycle of VD prior to RVD, 1 patient had 1 cycle of RD prior to RVD, 1 patient had 1 cycle of dexamethasone prior to RVD, 1 patient had 2 cycles of VD prior to RVD, and 1 patient had 2 cycles of RD prior to RVD).

Bortezomib toxicity requiring drug discontinuation occurred in $2(3 \%)$ patients; discontinuation was due to neuropathy in both cases. The number of cycles these patients received prior to bortezomib discontinuation is unknown. Lenalidomide toxicity requiring drug discontinuation occurred in 7 (12\%) patients after a median of 4 cycles (range 3-5); 3 (5\%) for venous thromboembolism, 2 (3\%) for rash, 1 (2\%) for recovery from surgery, and 1 (2\%) for unknown reasons. Peripheral 
neuropathy (any grade) was present in $30(50 \%)$ patients. Lenalidomide was held in the final cycle in 3 patients (5\%) in preparation for stem cell collection.

Table 1. Patient characteristics ( $\mathrm{N}=60)$. Median age at induction is 57 years with range of 39 to 71 years

\begin{tabular}{ll}
\hline Patient characteristic & $\mathbf{N}(\mathbf{\%})$ \\
\hline Male & $39(65)$ \\
Disease subtype & $30(50)$ \\
IgG & $13(21.7)$ \\
IgA & $16(26.6)$ \\
Light chain disease & $1(1.7)$ \\
Other & \\
ISS stage & $29(48)$ \\
Stage I & $18(30)$ \\
Stage II & $10(17)$ \\
Stage III & $3(5)$ \\
Unknown & \\
Cytogenetics & $38(63)$ \\
Standard & $15(25)$ \\
High & $7(12)$ \\
Unknown & \\
Number of RVD cycles & $3(5)$ \\
2 & $3(5)$ \\
3 & $13(22)$ \\
4 & $11(18)$ \\
5 & $13(22)$ \\
6 & $4(8)$ \\
7 & $11(18)$ \\
8 & $1(2)$ \\
10 & $1(2)$ \\
13 &
\end{tabular}

Table 2. Distribution of patients by best paraprotein response pre and post transplantation $(\mathrm{N}=60)$

\begin{tabular}{|c|c|c|c|c|}
\hline & \multicolumn{2}{|c|}{ Pre-ASCT response } & \multicolumn{2}{|c|}{ Post-RVD plus ASCT response } \\
\hline & N (\%) & $95 \%$ CI & N (\%) & $95 \% \mathrm{CI}$ \\
\hline $\mathrm{CR}$ & $30(50)$ & {$[37 \%, 63 \%]$} & $36(60)$ & {$[47 \%, 72 \%]$} \\
\hline VGPR & $16(26.7)$ & {$[16 \%, 40 \%]$} & $12(20)$ & {$[11 \%, 32 \%]$} \\
\hline PR & $11(18.3)$ & {$[10 \%, 31 \%]$} & $5(8)$ & {$[3 \%, 18 \%]$} \\
\hline SD & $0(0)$ & {$[0 \%, 6 \%]$} & $0(0)$ & {$[0 \%, 6 \%]$} \\
\hline PD & $1(2)$ & {$[0 \%, 9 \%]$} & $1(2)$ & {$[0 \%, 9 \%]$} \\
\hline Unknown & $2(3)$ & {$[0 \%, 12 \%]$} & $6(10)$ & {$[4 \%, 21 \%]$} \\
\hline
\end{tabular}

$\mathrm{CR}=$ complete paraprotein response, $\mathrm{VGPR}=$ very good paraprotein response; $\mathrm{PR}=$ partial paraprotein response, $\mathrm{SD}=$ stable disease; $\mathrm{PD}=$ progressive disease

Prior to ASCT, the rates of paraprotein CR, VGPR, and PR were 50\%, 26.7\%, and $18.3 \%$ respectively, while $2 \%$ and $3 \%$ had PD and missing values, respectively (see Table 2). The median time from RVD initiation to ASCT was 6.1 months (range: 3.9 to 21.8 months). The rate of treatment discontinuation due to drug-related toxicity was low, with $3 \%$ of patients discontinuing bortezomib and $12 \%$ discontinuing lenalidomide. 


\subsection{Stem cell collection and engraftment}

Stem cell collection was feasible after RVD and occurred at a median of 5.7 months (range: 3.6-21.6) from RVD initiation and at a median of 9.5 days (range: 6-105) prior to transplant.

The median number of CD34+ stem cells collected was $11.1 \times 10^{6} / \mathrm{kg}$ (range 2.8 to $75.4 \times 10^{6} / \mathrm{kg}$ ). A collection of more than $4 \times 10^{6} / \mathrm{kg}$ occurred in $58(97 \%)$ patients. The majority of patients $(\mathrm{N}=54(90 \%))$ were collected with cyclophosphamide plus filgrastim, $4(6.67 \%)$ patients were collected with filgrastrim alone, $1(1.67 \%)$ patient was collected with cyclophosphamide, filgrastim, and plerixafor, and $1(1.67 \%)$ patient had an unknown method of collection.

The number of cycles of RVD did not impact stem cell mobilization. Patients who received 6 or fewer cycles of RVD $(\mathrm{N}=43)$ collected a median of $11.0 \times 10^{6} / \mathrm{kg}$ (range 2.8 to $38.7 \times 10^{6} / \mathrm{kg}$ ) CD34+ stem cells while patients who received 7 or more cycles of RVD $(\mathrm{N}=17)$ collected a median of $11.2 \times 10^{6} / \mathrm{kg}$ (range 5.5 to $\left.75.4 \times 10^{6} / \mathrm{kg}\right) \mathrm{CD} 34+$ stem cells.

Median time to engraftment for neutrophils was 11 days (range 6 to 19) and for platelets was 19 days (range 10 to 92).

\subsection{Post-ASCT response}

Following ASCT, the rates of paraprotein CR, VGPR, and PR were $60 \%, 20 \%$, and $8 \%$ respectively, while $2 \%$ and $10 \%$ had PD and missing values, respectively (see Table 2).

Of the 30 patients with paraprotein CR pre-ASCT, $25(83 \%)$ patients remained in a paraprotein CR and $5(17 \%)$ patients had unknown disease status post-ASCT. Among the 30 patients who did not achieve a paraprotein CR prior to ASCT, 14 (47\%) patients improved their paraprotein response after ASCT, with 10 of these patients achieving a CR post-transplant (7 from pre-ASCT VGPR, 2 from PR, and 1 from PD), and 4 patients achieving a VGPR (all from pre-ASCT PR). In addition, 13 (43\%) of the 30 patients had no change in their response after ASCT, $1(3.2 \%)$ had PD and $2(6.7 \%)$ had unknown post-ASCT response status.

After ASCT, 42 (70\%) patients initiated lenalidomide maintenance starting a median of 110 days (range 55-465) from transplant.

\subsection{PFS following ASCT}

At median follow-up of 22.6 months, 11 patients have progressed and 2 patients have died ( 1 from their disease and 1 from angioimmunoblastic lymphoma 11 months post-ASCT without PD documented). The Kaplan-Meier estimate of PFS at 12 months post- ASCT is $90.3 \%$ (90\% CI: [81\%, 95\%]) (Figure 1, N=60).

We evaluated PFS according to risk status (ISS and cytogenetic status). From univariate results, the PFS at 12 months is 90\% (90\% CI: [71\%, 97\%]) and 92\% (90\% CI: [75\%, 97\%]) for high-risk ISS stages (2 and 3) and ISS stage 1 patients, respectively. The PFS at 12 months is $84 \%$ (90\% CI: [57\%, 95\%]) and 94\% (90\% CI: [81\%, 98\%]) for high risk and standard risk cytogenetics patients, respectively. No difference $(p>0.11)$ in progression-free survival was detected for ISS groups (stage II/III vs. stage I) or cytogenetics (high risk vs. standard risk) in univariate or multivariate models (see Table $3)$.

\section{Discussion}

Achievement of a deep response is an important and achievable objective of therapy in MM, as the ability to obtain a CR has consistently been associated with superior long-term treatment outcome ${ }^{[23]}$. RVD induction is associated with a high rate of both OR and CR. Whether ASCT consolidation improves the depth of response in patients treated with RVD induction is not known. 


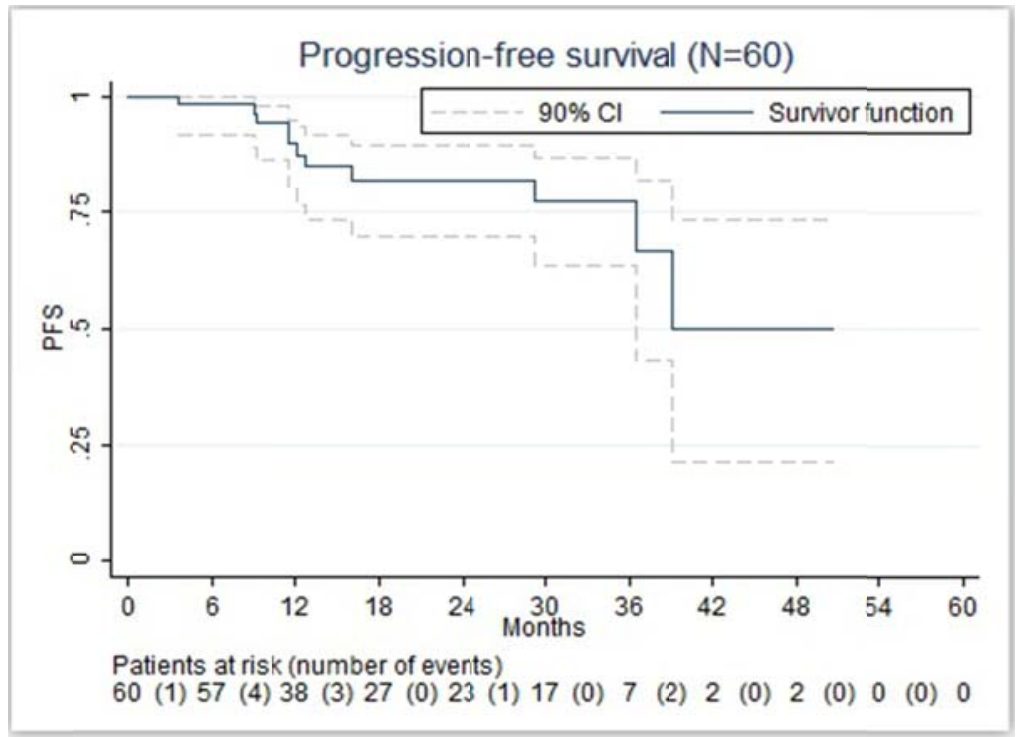

Figure 1. Kaplan Meier estimation of the progression-free survival. Patients who proceeded directly to ASCT after $\mathrm{RVD}$ induction $(\mathrm{N}=60)$

Table 3. Univariate and multivariable Cox proportional hazards regression models $(\mathrm{N}=60)$ to evaluate the effect of ISS and cytogenetic risk on progression free survival

\begin{tabular}{|c|c|c|}
\hline & Hazard ratio and $95 \%$ CI & $p$-value \\
\hline \multicolumn{3}{|l|}{ Univariate analysis } \\
\hline ISS (stage II/III vs. I ) & $1.62[0.43-6.11]$ & 0.478 \\
\hline $\begin{array}{l}\text { Cytogenetic risk (high risk vs } \\
\text { standard) }\end{array}$ & $2.76[0.78-9.76]$ & 0.116 \\
\hline \multicolumn{3}{|l|}{ Multivariable analysis } \\
\hline ISS (stage II/III vs. I ) & $1.34[0.34-5.27]$ & 0.680 \\
\hline $\begin{array}{l}\text { Cytogenetic risk (high risk vs } \\
\text { standard) }\end{array}$ & $1.10[0.28-4.35]$ & 0.889 \\
\hline
\end{tabular}

Our study demonstrates that ASCT consolidation improves the depth of response in patients treated with RVD induction. Among patients who did not achieve a paraprotein CR prior to ASCT, 47\% experienced an improvement in response post-transplant and all patients with a pre-transplant CR for whom post-ASCT data was available maintained their response.

Responses were durable, with a 12 month post-ASCT PFS rate of $90.3 \%$. It is noted that the PFS in our analysis reflects the impact of induction, ASCT, and maintenance therapy, since a majority of patients included in this analysis received lenalidomide maintenance.

Although CR is an important treatment objective in the management of MM, it serves only as a surrogate for the most clinically significant endpoints of PFS and OS. Whether ASCT following RVD induction extends PFS and OS relative to treatment with RVD alone is a critical question. This issue is the focus of an ongoing phase III clinical trial in which patients with newly diagnosed MM receive RVD induction followed by stem cell collection, with randomization to either immediate post-induction ASCT versus further conventional dose-therapy with RVD followed by lenalidomide maintenance. Patients in the latter arm have the option of ASCT at time of progression. 
Analysis of outcomes in high-risk MM subgroups - including ISS stage II/III disease or cytogenetic abnormalities associated with poor outcome - was limited in this study due to the relatively small sample size. We observed a trend towards less favorable outcomes in high-risk patients, however not to a level of statistical significance.

This study was limited by its retrospective nature, the relatively small number of patients, and the variation in frequency of pre-and post- transplant disease assessment inherent to a group of patients treated outside the context of a clinical trial. There is also potential selection bias due to inclusion criteria stipulating at least two cycles of RVD therapy. Finally, as previously noted, a significant number of patients received up to two cycles of non-RVD therapy consisting of VD, RD, or dexamethasone prior to RVD therapy.

Despite the limitations, this analysis does provide important data regarding treatment response associated with ASCT consolidation following RVD induction. It demonstrates that ASCT improved or maintained paraprotein response in patients who received RVD induction, and in particular improved the rate of CR.

\section{Acknowledgement}

The authors would like to thank Liana Langdon-Embry for her assistance in preparing the manuscript.

\section{References}

[1] Kyle R, Rajkumar SV, Plasma cell disorders, in Cecil textbook of medicine, L. Goldman and D.A. Ausiello, Editors. W.B. Saunders: Philadelphia; 2004: p. 1184-1195.

[2] Rajkumar SV, Blood E, Vesole D, Fonseca R, Greipp PR. Phase III clinical trial of thalidomide plus dexamethasone compared with dexamethasone alone in newly diagnosed multiple myeloma: a clinical trial coordinated by the Eastern Cooperative Oncology Group. J Clin Oncol. 2006; 24(3): 431-6. PMid:16365178 http://dx.doi.org/10.1200/JCO.2005.03.0221

[3] Rajkumar SV. Rosiñol L, Hussein M, et al. Multicenter, randomized, double-blind, placebo-controlled study of thalidomide plus dexamethasone compared with dexamethasone as initial therapy for newly diagnosed multiple myeloma. J Clin Oncol. 2008; 26(13): 2171-7. PMid:18362366 http://dx.doi.org/10.1200/JCO.2007.14.1853

[4] Zonder JA, Zonder JA, Crowley J, Hussein MA, et al. Lenalidomide and high-dose dexamethasone compared with dexamethasone as initial therapy for multiple myeloma: a randomized Southwest Oncology Group trial (S0232). Blood. 2010; 116(26): 5838-41. PMid:20876454 http://dx.doi.org/10.1182/blood-2010-08-303487

[5] Harousseau JL, Attal M, Avet-Loiseau H, et al. Bortezomib plus dexamethasone is superior to vincristine plus doxorubicin plus dexamethasone as induction treatment prior to autologous stem-cell transplantation in newly diagnosed multiple myeloma: results of the IFM 2005-01 phase III trial. J Clin Oncol. 2010; 28(30): 4621-9. PMid:20823406 http://dx.doi.org/10.1200/JCO.2009.27.9158

[6] Cavo M, Tacchetti P, Patriarca F, et al. Bortezomib with thalidomide plus dexamethasone compared with thalidomide plus dexamethasone as induction therapy before, and consolidation therapy after, double autologous stem-cell transplantation in newly diagnosed multiple myeloma: a randomised phase 3 study. Lancet. 2010; 376(9758): 2075-85. http://dx.doi.org/10.1016/S0140-6736(10)61424-9

[7] Richardson PG, Weller E, Lonial S, et al. Lenalidomide, bortezomib, and dexamethasone combination therapy in patients with newly diagnosed multiple myeloma. Blood. 2010; 116(5): 679-86. PMid:20385792 http://dx.doi.org/10.1182/blood-2010-02-268862

[8] Mitsiades N, Mitsiades CS, Poulaki V, et al. Apoptotic signaling induced by immunomodulatory thalidomide analogs in human multiple myeloma cells: therapeutic implications. Blood. 2002; 99(12): 4525-30. PMid:12036884 http://dx.doi.org/10.1182/blood.V99.12.4525

[9] Attal M, Harousseau JL, Stoppa AM, et al. A prospective, randomized trial of autologous bone marrow transplantation and chemotherapy in multiple myeloma. Intergroupe Francais du Myelome. N Engl J Med. 1996; 335(2): 91-7. PMid:8649495 http://dx.doi.org/10.1056/NEJM199607113350204

[10] Alvares CL, Davies FE, Horton C, et al. Long-term outcomes of previously untreated myeloma patients: responses to induction chemotherapy and high-dose melphalan incorporated within a risk stratification model can help to direct the use of novel treatments. Br J Haematol. 2005; 129(5): 607-14. PMid:15916682 http://dx.doi.org/10.1111/j.1365-2141.2005.05514.x 
[11] Alegre A, Díaz-Mediavilla J, San-Miguel J, et al. Autologous peripheral blood stem cell transplantation for multiple myeloma: a report of 259 cases from the Spanish Registry. Spanish Registry for Transplant in MM (Grupo Espanol de Trasplante Hematopoyetico-GETH) and PETHEMA. Bone Marrow Transplant. 1998; 21(2): 133-40. PMid:9489629 http://dx.doi.org/10.1038/sj.bmt.1701062

[12] Barlogie B, Tricot G, Anaissie E, et al. Thalidomide and hematopoietic-cell transplantation for multiple myeloma. N Engl J Med. 2006; 354(10): 1021-30. PMid:16525139 http://dx.doi.org/10.1056/NEJMoa053583

[13] Child JA, Morgan GJ, Davies FE, et al. High-dose chemotherapy with hematopoietic stem-cell rescue for multiple myeloma. N Engl J Med. 2003; 348(19): 1875-83. PMid:12736280 http://dx.doi.org/10.1056/NEJMoa022340

[14] Krejci M, Buchler T, Hajek R, et al. Prognostic factors for survival after autologous transplantation: a single centre experience in 133 multiple myeloma patients. Bone Marrow Transplant. 2005; 35(2): 159-64. PMid:15543200 http://dx.doi.org/10.1038/sj.bmt.1704728

[15] Lahuerta JJ, Martinez-Lopez J, Serna JD, et al. Remission status defined by immunofixation vs. electrophoresis after autologous transplantation has a major impact on the outcome of multiple myeloma patients. Br J Haematol. 2000; 109(2): 438-46. PMid:10848839 http://dx.doi.org/10.1046/j.1365-2141.2000.02012.x

[16] Nadal E, Giné E, Bladé J, et al. High-dose therapy/autologous stem cell transplantation in patients with chemosensitive multiple myeloma: predictors of complete remission. Bone Marrow Transplant. 2004; 33(1): 61-4. PMid:14704657 http://dx.doi.org/10.1038/sj.bmt.1704313

[17] O'Shea D, Giles C, Terpos E, et al. Predictive factors for survival in myeloma patients who undergo autologous stem cell transplantation: a single-centre experience in 211 patients. Bone Marrow Transplant. 2006; 37(8): 731-7. PMid:16501593 http://dx.doi.org/10.1038/sj.bmt.1705307

[18] Bjorkstrand B. Goldstone AH, Ljungman P, et al. Prognostic factors in autologous stem cell transplantation for multiple myeloma: an EBMT Registry Study. European Group for Bone Marrow Transplantation. Leuk Lymphoma. 1994; 15(3-4): $265-72$. PMid:7866274 http://dx.doi.org/10.3109/10428199409049723

[19] Lahuerta JJ, Mateos MV, Martínez-López J, et al. Influence of pre- and post-transplantation responses on outcome of patients with multiple myeloma: sequential improvement of response and achievement of complete response are associated with longer survival. J Clin Oncol. 2008; 26(35): 5775-82. PMid:19001321 http://dx.doi.org/10.1200/JCO.2008.17.9721

[20] Munshi NC, Anderson KC, Bergsagel PL, et al. Consensus recommendations for risk stratification in multiple myeloma: report of the International Myeloma Workshop Consensus Panel 2. Blood. 2011; 117(18): 4696-700. PMid:21292777 http://dx.doi.org/10.1182/blood-2010-10-300970

[21] Rajkumar SV, Harousseau JL, Durie B, et al. Consensus recommendations for the uniform reporting of clinical trials: report of the International Myeloma Workshop Consensus Panel 1. Blood. 2011; 117(18): 4691-5. PMid:21292775 http://dx.doi.org/10.1182/blood-2010-10-299487

[22] Kaplan E, Meier P. Nonparametric estimation from incomplete observations. J. Amer. Statist. Assn. 1958; 53: $457-481$. http://dx.doi.org/10.1080/01621459.1958.10501452

[23] Chanan-Khan AA, Giralt S. Importance of achieving a complete response in multiple myeloma, and the impact of novel agents. J Clin Oncol. 2010; 28(15): 2612-24. PMid:20385994 http://dx.doi.org/10.1200/JCO.2009.25.4250 